\title{
Survey of Poly Cystic Ovarian Disease (PCOD) Among The Girl Students of Bishop Heber College, Trichirapalli, Tamil Nadu, India
}

\author{
Nivetha.M and Susan .G.Suganya \\ P G Department of Zoology, Bishop Heber College (Autonomous), Tiruchirappalli-17, Tamil Nadu, India.
}

\begin{abstract}
Polycystic ovarian syndrome (PCOS) is a very common endocrine disorder encountered in women and is associated with problems, such as menstrual irregularities; hirsuitism; obesity; insulin resistance; acne; and, in later life, infertility with diabetes mellitus and uterine cancer. Early diagnosis is necessary for early interventions to minimize the immediate and chronic consequences. The prevalence of PCOS among the girl students has not yet been clearly determined. This study is an attempt to assess its prevalence in the girl students of a college. We undertook a survey among the female students of Bishop Heber College (Autonomous), Trichirappalli City of Tamil Nadu. Since students from all parts of India study here, the study population represents a random sample of female students (252 Students) in the college and age group was between 18-31yrs. The study period was five months from November 2015 to March 2016. PCOS was diagnosed by using a questionnaire with Rotterdam's criteria and the prevalence was found to be $7.14 \%$. This study definitely created awareness among the adolescent college girls about PCOS. This will help them to modify their life style and to have better reproductive life later. During this study we identified the adolescents with risk for developing PCOS, we ask them to take proper diagnosis and treatment with consultation of a gynecologist. In married girl students, especially in the presence of other risk factors for infertility, early conception is advised and to find the effectiveness of awareness programme.
\end{abstract}

Keywords: PCOD, PCOS, Menstrual abnormalities, 'Stein-Leventhal Syndrome', 'Syndrome O.

\section{Introduction}

The term Polycystic Ovarian Disease (PCOD) was first described by Irving Stein and Michael Leventhal as a Triad of 'Amenorrhoea', 'Obesity' and 'Hirsutism' In 1935 when they observed the relation between obesity and reproductive disorders.[1].It is hence also known as the 'Stein-Leventhal Syndrome' or 'Hyperandrogenic Anovulation' (HA)and is the most common endocrine ovarian disorder affecting approximately 2-8\% women of reproductive age worldwide.[2] Now a days, it is also referred to as the 'Syndrome O' i.e. Overnourishment, Overproduction of insulin, Ovarian confusion and Ovulatory disruption. So, PCOD is called as Polycystic Ovarian Syndrome (PCOS).

PCOS is currently considered as a lifestyle disorder affecting $2.2-26 \%$ of young girls in their reproductive age in India. A study conducted on 460 girls in the age group of 15-18 years from a residential college of Andhra Pradesh reported a prevalence of 9.13\% in Indian adolescents.[3] Though globally it has an alarming incidence, its diagnosis is difficult as it manifests as a spectrum of symptoms than a specific one. It is primarily characterized by an extremely irregular menstrual cycle in which ovulation may not occur.[4].Normal pubertal events include Oligomenorrhoea, Hirsutism, Acne and Weight gain. However, no single criterion is sufficient for clinical diagnosis due to multiple aetiologies and presentations.[4].Paediatric Endocrinologists nowadays tend towards an earlier work-up instead of the traditional practice of waiting for two years postmenarche.[5]

PCOS is a common disorder, often complicated by chronic anovulatory infertility and hyperandrogenism with the clinical manifestation of oligomenorrhoea, hirsutism and acne.[6] Many women with this condition are obese and have a higher prevalence of impaired glucose tolerance, type 2 diabetes and sleep apnoea than is observed in the general population. They exhibit an adverse cardiovascular risk profile, characteristic of the cardiometabolic syndrome as suggested by a higher reported incidence of hypertension, dyslipidaemia, visceral obesity, insulin resistance and hyperinsulinaemia.[7]

PCOS is frequently diagnosed by gynaecologists and it is therefore important that there is a good understanding of the long-term implications of the diagnosis in order to offer a holistic approach to the disorder. The calculation of the prevalence of PCOS depends on the criteria used to define this syndrome. Because the symptoms of PCOS emerge insidiously and are coincident with changes that accompany normal pubertal development, subtle features may not be realized in the early stages; this may account for the failure to identify the disorder in young girls. 
It is possible to identify the early clinical manifestations of PCOS in late puberty and early adolescence. The disease is on its rise because of lifestyle and environmental changes occurring with modernization. Initially, the condition is asymptomatic in young women, progressing to produce menstrual irregularities, excessive weight gain, and infertility; eventually, in late middle age, it leads to several major health hazards. Early diagnosis is necessary for early intervention, including behavior modification, to minimize the immediate and chronic consequences of PCOS. Polycystic ovary syndrome is the most common hormone disorder in women, affecting 5 percent to 10 percent of adolescent girls and adult women of child-bearing age. The signs of PCOS include excessive hair growth on the face and abdomen, acne, irregular or absent menstrual periods, failure of ovulation, and reduced fertility. PCOS usually begins at or soon after puberty and is a lifelong condition. Obesity is present in 50 percent of individuals with PCOS. In addition, women with PCOS are at increased risk of developing diabetes, cardiovascular disease, obstructive sleep apnea, and cancer of the uterus.

In view of this and the fact that the prevalence of this syndrome in our community remains unknown, we attempted to find its prevalence in a community of women aged between 18-31 years.

\subsection{The main objectives of this study were to}

1) Identify the adolescent girls who are at high risk for PCOS using various symptoms include:

- Missed periods, irregular periods, or very light periods

- Ovaries that are large or have many cysts

- Excess body hair, including the chest, stomach, and back (hirsutism)

- Weight gain, especially around the belly (abdomen)

- Acne or oily skin

- Male-pattern baldness or thinning hair

- Infertility

- Small pieces of excess skin on the neck or armpits (skin tags)

- Dark or thick skin patches on the back of the neck, in the armpits, and under the breasts

- Acanthosis nigricans(Acanthosis nigricans is a brown to black, poorly defined, velvety hyperpigmentation of the skin.) may present with thickened, velvety, relatively darker areas of skin on the neck, armpit and in skin folds.

2) Find association between PCOS risk status with selected variable i.e. general health status. PCOS is a common disorder, often complicated by chronic anovulatory infertility and hyperandrogenism with the clinical manifestation of oligomenorrhoea, hirsutism and acne.

3) Many women with this condition are obese and have a higher prevalence of impaired glucose tolerance, type2diabetes and sleep apnoea than is observed in the general population. They exhibit an adverse cardiovascular risk profile, characteristic of the cardio metabolic syndrome as suggested by a higher reported incidence of hypertension, dyslipidaemia, visceral obesity, insulin resistance and hyperinsulinaemia.

4) PCOS is frequently diagnosed by gynaecologists and it is therefore important that there is a good understanding of the long-term implications of the diagnosis in order to offer a holistic approach to the disorder. Hence refer the girls who have PCOS to the gynecologists.

5) To make an awareness about polycystic ovary syndrome (PCOS) among adolescent college girls.

The major purpose of this study is to create awareness among adolescent college girls about PCOS. This will help them to modify their life style and to have better reproductive life later.

\subsection{Prevalence of PCOS}

Estimation of the 'true' prevalence has to be made with caution as many of the data available were collected prior to the new Rotterdam diagnostic criteria. Most clinical data suggests a prevalence of 6-7\% of the population.[5-8].The present Rotterdam criteria are current best practice but it is recognized that PCOS encompasses a wide spectrum of disorder, overlapping with normality. The prevalence of PCOS may differ according to ethnic background; for example, in women of South Asian origin, PCOS presents at a younger age, has more severe symptoms and a higher prevalence $[9,10]$

\subsection{Risk factors of PCOS}

\subsubsection{Cardiovascular Disease}

In January 1997 researchers in New Zealand reported that women with multiple cysts on their ovaries were at increased risk of heart disease. In the study $42 \%$ of women with heart disease also had 8/> ovarian cysts compared to $22 \%$ of women without heart disease. They should hence be regularly monitored and advised to consume less fat and cholesterol.Similarly, PCOS is characterized by endothelial dysfunction and resistance to the vasodilating action of insulin.[11] An increased risk of myocardial infarction in PCOS women than agematched controls has also been reported.[12] 
Survey of Poly Cystic Ovarian Disease (Pcod) Among The Girl Students of Bishop Heber College,..

\subsubsection{Obesity}

Obesity is also a feature observed and estimated to effect 50\% of PCOS women[13], classically presented in patients with upper body obesity which has been associated with menstrual disturbances [14].It amplifies biochemical and clinical abnormalities of PCOS.[15].Previously obesity was thought to be the cause of PCOS but now understood as a modifier of the condition.

\subsubsection{Infertility}

Another complicating feature of PCOS is the effects it has on ovulation and fertility with $>75 \%$ of women with anovulation infertility [16] and treatment is based upon the patient ${ }^{\text {te }} \mathrm{s}$ characteristics. Besides follicular arrest and impaired selection of dominant follicle is possible [17] as also the risk of multiple pregnancy with treatment. [18]

\subsection{Complications of PCOS}

\subsubsection{Endometrial cancer}

The long-term follow-up of 786 PCOS women found an increased risk of endometrial cancer.[19].A study revealed that in women $>50$ years of age with endometrial cancer $62.5 \%$ showed a greater prevalence of PCOS than $27.33 \%$ who were not suffering from the syndrome.(P=0.033)[20].

\subsubsection{Complications in pregnancy}

Women with PCOS have a greater risk of complications viz. Gestational diabetes [21], pre-eclampsia, pre-term labour, small for gestational age, [22]pregnancy induced hypertension (PIH),[23] spontaneous abortions [24]etc.

\subsubsection{Sleep Apnoea}

It has been reported that women with PCOS have increased Sleep Disordered Breathing (SDB) and daytime sleepiness.[25]

\subsubsection{Depression}

There was a higher prevalence of depression in PCOS patients associated with higher body mass index (BMI, $\mathrm{P}=0.05)$ and greater insulin resistance $(\mathrm{P}=0.02)$ [26].Besides, Impaired Glucose Tolerance[27], Dyslipidaemia, Metabolicsyndrome, Non-alcoholic steatohepatitis and higher levels of C-reactive protein, a disease marker for cardiovascular diseases are other probable risks involved.

\section{Materials and Methods}

The medical history was recorded, with Anthropometric, clinical, and biochemical parameters by using the questionnaire on whether the participant had been previously diagnosed with PCOS. A few questions were included to exclude thyroid disorders, hyper prolactinemia, and late-onset congenital adrenal hyperplasia. Menstrual irregularity was assessed as a usual cycle length of less than 21 days or more than 35 days. Clinical hyperandrogenism was assessed on the basis of the self-reported degree of hirsutism by using self assessment method. Results were kept confidential. The selected participants likely to have PCOS asked to go for further clinical and ovarian ultrasound examination.

A positive finding of polycystic ovaries was defined by the detection of 12 or more follicles measuring 2-9 $\mathrm{mm}$ in diameter and/or increased ovarian volume of above $10 \mathrm{~cm} 3$ in at least one of the ovaries. PCOS was defined by the presence of 2 or more of the following three Rotterdam criteria in addition to the exclusion of related disorders (ESHRE/ASRM (Rotterdam) 2003 criteria (European Society for Human Reproduction and Embryology/American Society for Reproductive Medicine).

This is a questionnaire based study on the awareness of polycystic ovary syndrome. The participants who undertook the survey are undergraduate and postgraduate girl students of a college. A total of 25 questions were asked to 252 girl students from various departments in the college and age group was between 18-31yrs. Individuality was assured when the subjects filled the survey. The questionnaire is filled in paper and pen method. After the data collection, statistical measurements are done. The questions included are:

\section{The Questionnaire}

Anthropometric, clinical, and biochemical parameters

(1) Age yrs

(2) Height $\mathrm{cms}$

(3) Weight kg

(4) Body Mass Index (BMI)

(5) Waist circumference $\mathrm{kg} / \mathrm{m}^{2}$ (calculated) (cm) 
(6) Hip circumference $(\mathrm{cm})$

(7) Age of first period

(8) Regularity of the menstrual cycle

(9) Pelvic pain during menstruation

(10) Marital status If Married

(11) Fertility problems

(12) Use of oral contraceptive Pills

(13) Presence of PCOD If Yes, Answer the following

(14) Diet

(15) Obesity

(16) Blood pressure___ (mmHg)

(17) Serum FSH and LH level

(18) Serum Testosterone level and $(\mathrm{ng} / \mathrm{dl})$ $(\mathrm{ng} / \mathrm{dl})$

(19) Hirsutism (Unwanted hair growth) of face and body

(20) Acne

(21) Stretch marks (Stria)

(22) Hair loss

(23) Acanthosis nigricans (Skin disorder)

(24) Other systemic disorders

Diabetes , Hyperthyroidism , Hypertension High Cholesterol __ Depression and mood change (25) Family History: Your Mother with PCOD , Stress , Your siblings with PCOD

\subsection{Limitations of the study}

This study does have some limitations. Menstrual diaries to identify menstrual intervals were not utilized. The patients were not evaluated for the presence of biochemical hyperandrogenemia. Mid-luteal progesterone level was not measured to identify eumenorrhoeic women, who had sub-clinical menstrual dysfunction. Since the study was performed in students, the majority of the subjects unmarried, which precluded a vaginal approach for ultrasonography and as a result, this specific and sensitive tool was not used for identifying polycystic ovaries.

\section{Results and Discussion}

The study population comprised about 252 girls from various departments. The study reveals that among 67 girls of $17-19$ years age group 3 of them were with symptoms of PCOD. About 71 girls of $20-22$ years age group 8 girls were with symptoms of PCOD. About 47 girls of $23-25$ years age group 10 girls were with symptoms of PCOD. About 39 girls of $26-28$ years age group 5 girls were with symptoms of PCOD. About 28 girls of 29 - 31 years age group 2 girls were with symptoms of PCOD (Table-VI).

Totally 43 girls (Table-I) in different age group were found to be having irregular menstrual cycles. 28 girls were detected with polycysts in their ovaries (Table-IV) and 28 girls were found to be with hirsuitism (Table-II). Among the girls studied about 46 of them were found to have increased acne (Table-III). The mean BMI of girls in age groups $17-19,20-22,23-25,26-28$ and $29-31$ were 23.4, 21.1, 23.5, 24.2 and 23.5 respectively(Table-V).

PCOS is the most common endocrine abnormality in reproductive- age women. The prevalence of PCOS is traditionally estimated at $4 \%$ to $8 \%$ from studies performed in Greece, Spain and the USA [1-4]. The prevalence of PCOS has increased with the use of different diagnostic criteria and has recently been shown to be $18 \%(17.8 \pm 2.8 \%)$ in the first community-based prevalence study based on current Rotterdam diagnostic criteria [5]. USG Criteria for diagnosis of PCOS are presence of 12 or more follicle in one ovary arranged peripherally in necklace pattern, each follicle measures $2-9 \mathrm{~mm}$ in diameter and ovarian volume $>10 \mathrm{~cm}$ cube. Laparoscopic criteria include enlarged pearly white ovaries with multiple follicles. Endocrine abnormalities associated with PCOS are elevated LH, normal FSH and LH/FSH ratio > 3:1. Long term consequences of PCOS include metabolic syndrome like diabetes mellitus, dyslipidemia and coronary heart disease .Women with PCOS are prone for development of endometrial and breast cancer in later life [5].

Table-I : Total Number Of Students Having Irregular Periods

\begin{tabular}{|c|c|c|c|c|}
\hline S.NO & AGE & $\begin{array}{c}\text { TOTAL NO OF } \\
\text { STUDENTS } \\
\text { SURVEYED }\end{array}$ & $\begin{array}{c}\text { TOTAL NO STUDENTS } \\
\text { HAVING } \\
\text { IRREGULAR PERIODS }\end{array}$ & $\begin{array}{c}\text { \% OF STUDENTS } \\
\text { WITH PCOS }\end{array}$ \\
\hline 1 & $17-19$ & 67 & 13 & 19.4 \\
\hline 2 & $20-22$ & 71 & 12 & 16.9 \\
\hline 3 & $23-25$ & 47 & 13 & 27.65 \\
\hline 4 & $26-28$ & 39 & 3 & 7.69 \\
\hline 5 & $29-31$ & 28 & 2 & 7.14 \\
\hline
\end{tabular}




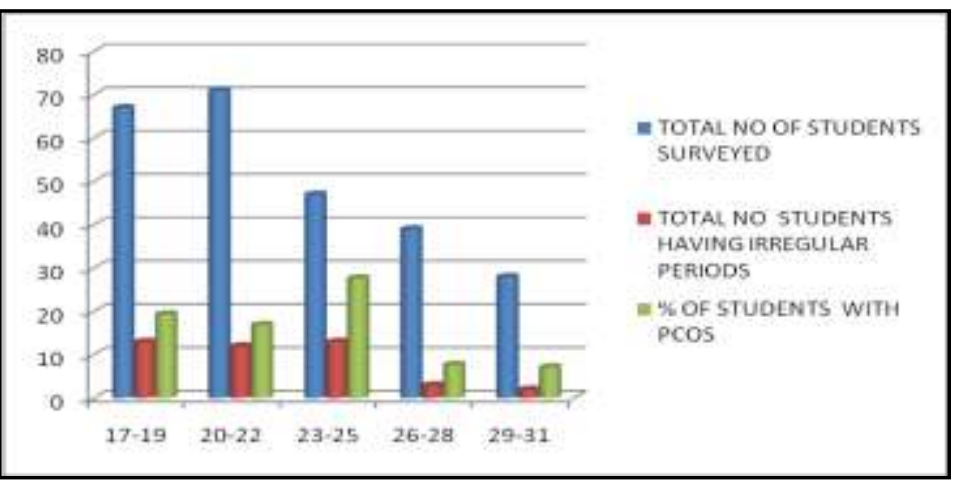

Fig -1

Table-II : Total Number Of Students Having Hirsutism

\begin{tabular}{|c|c|c|c|c|}
\hline S.NO & AGE & $\begin{array}{c}\text { TOTAL NUMBER OF } \\
\text { STUDENTS }\end{array}$ & $\begin{array}{c}\text { TOTAL NO.OF STUDENTS } \\
\text { HAVING HIRSUTISM }\end{array}$ & $\begin{array}{c}\text { \% OF STUDENTS } \\
\text { HAVING HIRSUTISM }\end{array}$ \\
\hline 1 & $17-19$ & 61 & 3 & 4.47 \\
\hline 2 & $20-22$ & 71 & 8 & 11.26 \\
\hline 3 & $23-25$ & 47 & 10 & 21.27 \\
\hline 4 & $26-28$ & 39 & 5 & 12.82 \\
\hline 5 & $29-31$ & 28 & 2 & 7.14 \\
\hline
\end{tabular}

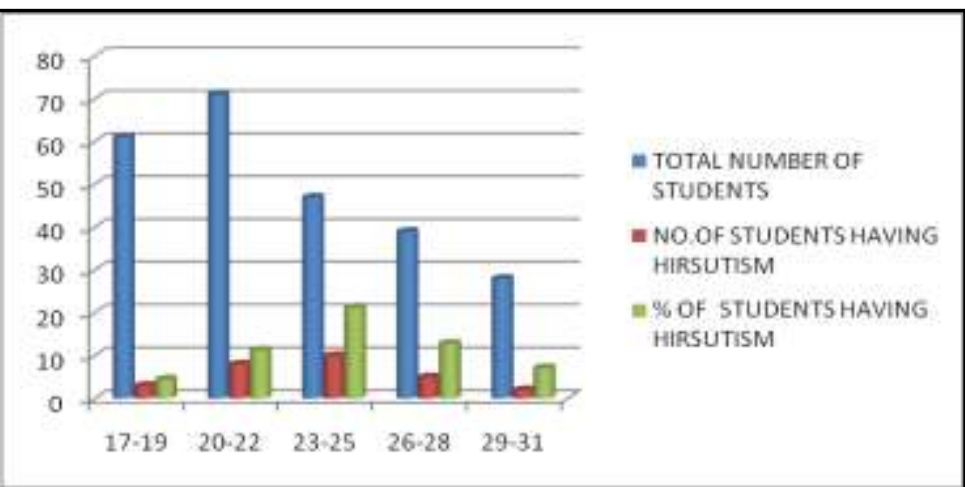

Fig -2

Table-III : Total Number Of Students Having Incresed Acne

\begin{tabular}{|c|c|c|c|c|}
\hline S.NO & AGE & $\begin{array}{c}\text { TOTAL NO.OF } \\
\text { STUDENTS }\end{array}$ & $\begin{array}{c}\text { TOTAL NO STUDENTS } \\
\text { HAVING INCRESED ACNE }\end{array}$ & $\begin{array}{c}\text { \% OF STUDENTS HAVING } \\
\text { INCRESED ACNE }\end{array}$ \\
\hline 1 & $17-19$ & 67 & 11 & 16.41 \\
\hline 2 & $20-22$ & 71 & 16 & 22.53 \\
\hline 3 & $23-25$ & 47 & 12 & 25.53 \\
\hline 4 & $26-28$ & 39 & 6 & 15.38 \\
\hline 5 & $29-31$ & 28 & 1 & 3.57 \\
\hline
\end{tabular}

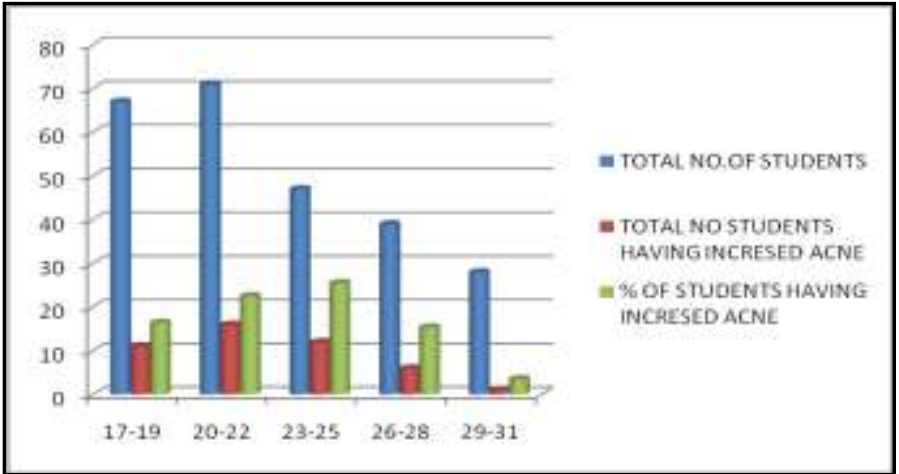

Fig -3 
Survey of Poly Cystic Ovarian Disease (Pcod) Among The Girl Students of Bishop Heber College,..

Table-IV : Total Number Of Students With PCOS

\begin{tabular}{|c|c|c|}
\hline S.NO & AGE & TOTAL NO. OF STUDENTS WITH PCOS \\
\hline 1 & $17-19$ & 3 \\
\hline 2 & $20-22$ & 8 \\
\hline 3 & $23-25$ & 10 \\
\hline 4 & $29-28$ & 5 \\
\hline 5 & $29-31$ & 2 \\
\hline
\end{tabular}

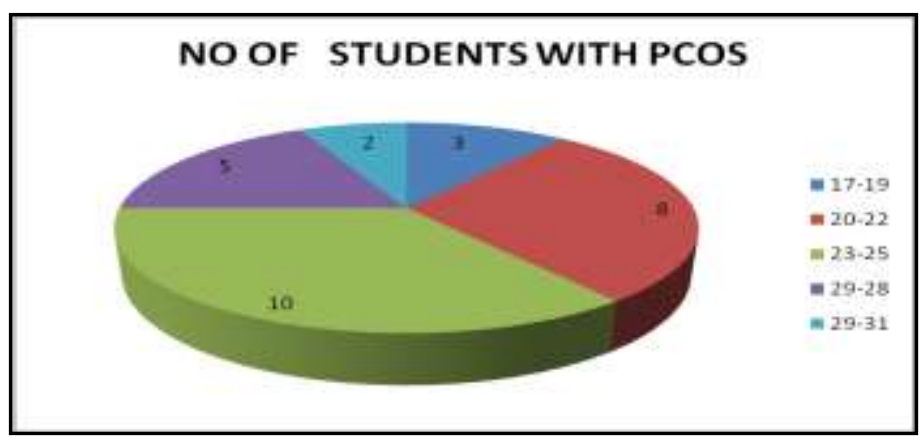

Fig -4

Table-V: Mean Height $(\mathrm{Cm})$, Mean Weight $(\mathrm{Kg})$, Body Mass Index

\begin{tabular}{|c|c|c|c|c|}
\hline S.NO & AGE & MEAN HEIGHT (cm) & MEAN WEIGHT(kg) & BMI \\
\hline 1 & $17-19$ & 144.03 & 48.5 & 23.4 \\
\hline 2 & $20-22$ & 154.58 & 50.32 & 21.1 \\
\hline 3 & $23-25$ & 149.33 & 52.37 & 23.5 \\
\hline 4 & $26-28$ & 152.32 & 56.23 & 24.2 \\
\hline 5 & $29-31$ & 150.28 & 53 & 23.5 \\
\hline
\end{tabular}

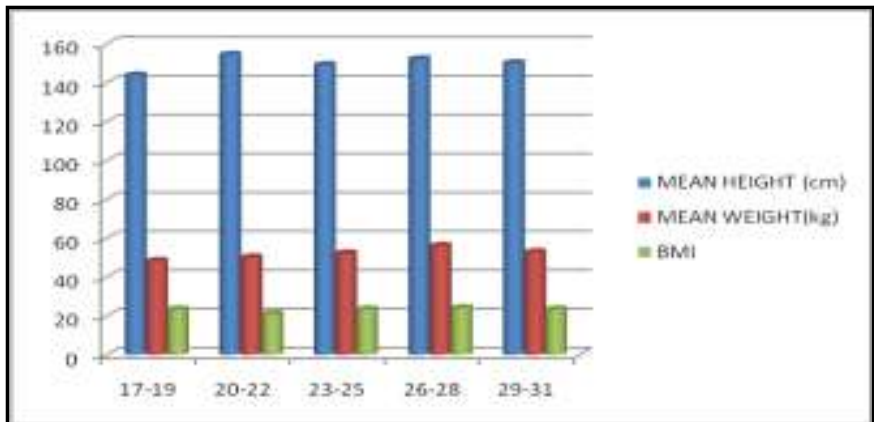

Fig -5

Table-VI : Number Of Students Identified PCOS

\begin{tabular}{|c|c|c|c|c|}
\hline S.NO & AGE & $\begin{array}{c}\text { TOTAL NO.OF STUDENT } \\
\text { SURVEYED }\end{array}$ & $\begin{array}{c}\text { NO.OF STUDENTS } \\
\text { IDENTIFIED PCOS }\end{array}$ & $\begin{array}{c}\text { \%OF STUDENTS } \\
\text { WITH PCOS }\end{array}$ \\
\hline 1 & $17-19$ & 67 & 3 & 4.48 \\
\hline 2 & $20-22$ & 71 & 8 & 11.26 \\
\hline 3 & $23-25$ & 47 & 10 & 21.28 \\
\hline 4 & $26-28$ & 39 & 5 & 12.82 \\
\hline 5 & $29-31$ & 28 & 2 & 7.14 \\
\hline
\end{tabular}

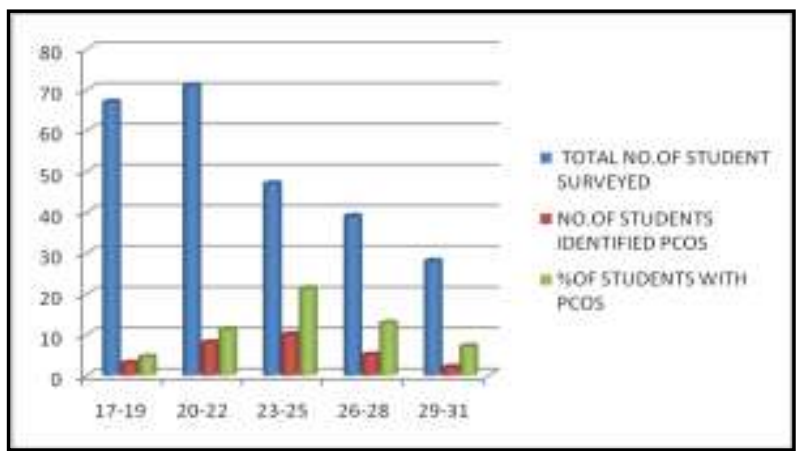

Fig -6 
Results indicated that there are $7.14 \%$ (Table-VI) of surveyed girls were having PCOS and the identified adolescents with risk for developing PCOS, we ask them to take proper diagnosis and treatment with consultation of a gynecologist for treatment for acne, hyperandrogenism, and menstrual abnormalities. However, the primary treatment is a healthy diet and regular exercise. Implications for practice included; a need for increased awareness, early recognition, comprehensive screening, prompt treatment, and frequent follow-ups for all PCOS adolescents to prevent future morbidities. In married girl students, especially in the presence of other risk factors for infertility, early conception is advised. The final implication was the need for more research involving adolescent females with PCOS.The crude prevalence rate determined from this study was $17 \%$. The findings of this study revealed that PCOS occurs in about 3 of 20 women. This once again proved that PCOS is the most common endocrine abnormality among women in the reproductive and adolescent age groups.

Among the patients with PCOS, 70\% had oligomenorrhoea and polycystic ovaries, 13.3\% had hirsutism and polycystic ovaries, and 3.3\% had oligomenorrhoea and hirsutism. Another $13.3 \%$ had all the 3 features. Thus, the majority of the patients had oligomenorrhoea with polycystic ovaries, which stresses the importance of the proper evaluation of young girls presenting with irregular cycles or excessive hair growth before considering them as normal changes associated with pubertal development.

Obesity aggravates this alone or in combination with other factors. In 2005, reports showed that lifestyle changes in both rural and urban areas have influenced the prevalence of PCOS.[28] Further, Studies showed that lack of exercise and low physical activity play a role in the prevalence of PCOS.[29]. Long-term preventive measures should be implemented early. We recommend that an optimal body mass index of $<27$ and $\mathrm{W} / \mathrm{H}$ ratio of $<0.8$ be maintained.

Various lifestyle modifications, including regular exercise half hour daily or one hour on alternate days and dietary modifications in the form of small frequent meals will be required to achieve weight reduction. Proper evaluation and early diagnosis should be done in adolescents presenting with features of PCO. In married girl students, especially in the presence of other risk factors for infertility, early conception is advised.

The prevalence of PCOS is traditionally estimated at $4 \%$ to $8 \%$ from studies performed in Greece, Spain and the USA. [30-33]. Our study is also proved that 7.14\% are identified with PCOS in the age of 18-31 yrs. It is coincides with the exploratory survey to identify the adolescents with high risk for Polycystic Ovarian Syndrome (PCOS) and to find the effectiveness of an awareness programme among students of selected pre university colleges of Udupi District [34]. Likewise our study is also to identify the adolescents with risk for developing PCOS and to find the effectiveness of awareness programme.

Seventy five percent of women suffering from PCOS were in between the age group of 19 to 26 years and ten percent were below 19 years of age. This was also proved in our study. This could be attributed to the age at marriage in rural area and subsequent investigations for evaluation of infertility. Eighty percent of women were unmarried and only twenty percent were married at the time of investigation. During investigation unmarried girls presented mainly with complaints of menstrual abnormalities like oligo and or hypomenorrhoea. Remaining cases had isolated secondary amenorrhoea. In married girls the duration of married life was less than two years at the time of investigation.[35]

Thirty five percent women were either overweight or obese at the time of diagnosis. Only ten percent women belonged to lean PCOS category. Eighty to eighty five percent cases had Ultrasonographic and endocrinal evidence of PCOS. Weight reduction and life style modification were advised to all women having abnormal BMI values.

Most of the unmarried girls and few recently married girls with menstrual abnormalities were asked to take treatment with the consultation of gynecologist.In our study we identified 43 (i.e:17\%) students with irregular periods. It has been known for many years that severe oligo- and amenorrhoea in the presence of premenopausal levels of estrogen can lead to endometrial hyperplasia and carcinoma. In women with PCOS intervals between menstruations of more than 3 months may be associated with endometrial hyperplasia.

Women who are oligomenorrhoeic and do not have normal withdrawal bleeds should be investigated and send for the consultation of gynecologist. This may include ultrasound scan, endometrial sampling and/or hysteroscopy. In our study we identified 8 students with obese out of 28 PCOS cases. Lifestyle changes through diet and exercise remain the first line for treatment of obesity in PCOS. PCOS is often associated with obesity and abnormal fat distribution, especially of abdominal fat, even where the BMI is normal. Obesity worsens insulin resistance that may exacerbate this dysfunction [36].

In our study we identified 28 (i.e: $11 \%$ ) students with hirsutism. Hirsutism in the setting of PCOS is difficult to treat and there are currently no large randomized control trials on its treatment in this patient group. Licensed treatments for hirsutism include oral contraceptive pills, dianette (oestrogen and cyproterone acetate), cosmetic measures (such as laser, electrolysis, bleaching, waxing and shaving) and topical facial eflornithine. However, there is a paucity of good-quality robust placebo controlled trials for hirsutism treatment, particularly for combination therapy. 
Lifestyle alteration will reduce the likelihood of developing type 2 diabetes later stages in life. Effects of hyperandrogenisation are among the most deleterious long-term consequences of PCOS when taken into consideration of its impact on a woman's self-image perception and the subsequent psychological effects.

\section{Conclusion}

In conclusion, PCOS is a common endocrine disorder of female adolescence and adulthood with exact etiology unknown but pathophysiology rooted in insulin resistance, hyperandrogenism, and chronic anovulation. A multitude of clinical factors can present including hirsutism, menstrual irregularities, metabolic abnormalities, acne, and increased BMI. History, physical exam, and laboratory tests are all components of making a diagnosis as some adolescents do not present with all clinical factors. Treatment options include healthy dietary habits and regular exercise accompanied by additional medications to treat presenting symptoms.

Primary care providers should be suspicious of PCOS in adolescent females regardless of their weight. A high level of suspicion will facilitate timely recognition, prompt treatment, and potential reduction or elimination of future morbidities until more information is known. This study will explore the complex mechanisms behind the manifestation of PCOS and how these, and other factors, may make diagnosis difficult. In order to thoroughly understand these aspects of PCOS, the causes, symptoms, and treatments were explored. The diversity of causes and symptoms of the disease add to the difficulty in diagnosis and treatment of PCOS. The best treatment option varies among individuals, and the effectiveness of each treatment can vary according to an individual's condition. The research on all of these aspects of PCOS will inform the public about how PCOS can affect them and their loved ones. From this newly gathered knowledge, individuals may be able to protect themselves from some of the negative consequences associated with PCOS.

\section{Acknowledgements}

Our grateful thanks are due to the Principal, HOD and other Staff members of Zoology Department, Bishop Heber College (Autonomous), Trichirappalli for the permission, many helpful suggestion, support, constructive advice and encouragement rendered throughout for the completion of this work. Our heartfelt thanks to all the girls participating in this study; thank you for your positive attitude, trust and patience.

\section{References}

[1]. SteinIF,Leventhal MN.Amenorrhoea associated with bilateral Polycystic Ovaries. American Journal of Obstetrics and Gynaecology 1935; 29:181.

[2]. Knochenhauer ES.et al. Prevalence of Polycystic Ovarian Syndrome. Journal of Clinical Endocrinology \&Metabolism1998; 83(9):3068-3082.

[3]. Nidhi R et al. Prevalence of Polycystic Ovarian Syndrome in Indian adolescents. Journal of Paediatric Adolescent Gynaecology 2011 Aug;24(4):223-227.

[4]. Azziz R et al. The prevalence and features of Polycystic Ovarian Syndrome in an unselected population. Obstetrics and Gynaecology 2004Jun;89(6):2745-2749.

[5]. Guttmann-Bauman I.The prevalence and features of Polycystic Ovarian Syndrome in paediatric endocrine community in the USA . Journal of PaediatricEndocrinology\& Metabolism,2005

[6]. Franks S. Polycystic ovary syndrome. N Engl J Med. 1995;333:853-61

[7]. Glueck CJ, Papanna R, Wang P, Goldenberg N, Sieve-Smith L. Incidence and treatment of metabolic syndrome in newly referred women with confirmed polycystic ovarian syndrome. Metabolism. 2003;52:908-15.

[8]. Rotterdam ESHRE/ASRM sponsored PCOS consensus workshop group, Revised 2003 consensus on diagnostic criteria and longterm health risks to PCOS. Fertil Steril 2003;81:19-25.

[9]. Rodin DA, Bano G, Bland JM, Taylor K, Nussey SS. Polycystic ovaries and associated metabolic abnormalities in Indian subcontinent Asian women. Clin Endocrinol (Oxf) 1998;49:91-9.

[10]. Wijeyaratne CN, Balen AH, Barth JH, Belchetz PE. Clinical manifestations and insulin resistance (IR) in polycystic ovary syndrome (PCOS) among South Asians and Caucasians: is there a difference? Clin.Endocrinol (Oxf) 2002;57:343-50.

[11]. ParadisiG etal.Polycysticovarian syndromeisassociatedwithendothelialdysfunction.Circulation.2001; 103:1410-1415.

[12]. Dahlgren E et al.Polycystic ovarian syndrome and risk for myocardial infarction. Acta Obstetricia et Gynecologicaandinavica. 1992;71:599-603.

[13]. GoldzieherJW, Young RL.Selected aspects of polycystic ovarian disease. Endocrinology Metabolism Clinics of North America. 1993Mar;21(1):141-171.

[14]. Hartz et al. The association of obesity with infertility and related menstrual disturbances.InternationalJournalofObesity.1979; 3:57-73.

[15]. Kiddy.DS et al. Improvement in endocrine and ovarian function during dietary treatment of obese women with PCOS. Clinical Endocrinology.(Oxford)1992;36:105-111.

[16]. HomburgR.The management of infertility associated with Polycystic ovarian syndrome. Reproductive Biology and Endocrinology.2003;1:109

[17]. Webber LJ et al.Formationandearlydevelopmentoffolliclesinthepolycystic ovaries.Lancet.2003; 362:1017-1021.

[18]. Jonard S, Dewailly D. The follicular excess in polycystic ovaries due to intraovarian hyperandrogenism may be the main culprit for follicular arrest.Human Reproduction Update.2004;10(2):107-117.

[19]. Wild $\mathrm{S}$ et al.Card iovas culard isease inwom en with polycy sticovarian syndrom eatlong-term followup: aretros pectiveco hortstudy.Human Fertility.2000;52:595-600.

[20]. Pillay OC et al.Theassociationbetweenpolycysticovariesandendometrialcancer.HumanReproduction.2006;21:924-929.

[21]. Bjercke S et al. Impact of insulin resistance on pregnancy complications and outcomes in women with polycystic ovarian syndrome. Gynaecologic and ObstetricInvestigation.2002;54:94-98. 
[22]. Petermann $\mathrm{T}$ et al.Birth weight in offspring of mothers with polycystic ovarian syndrome.Human Reproduction.2005 Aug;20(8):2122-2126.

[23]. Weerakiet S et al.Prevalence of gestational diabetes mellitus and pregnancy outcomes in Asian women with polycystic ovary syndrome.Gynecological Endocrinology.2004 Sep;19(3):134-140.

[24]. Wang JX et al.Polycystic ovarian syndrome and the risk of spontaneous abortion following assisted reproductive technology treatment.Human Reproduction.2001Dec;16(12):2606-2609.

[25]. VgontzasAN et al.Sleep apnoea is a manifestation of the metabolic syndrome.Sleep Medicine Reviews.2005 Jun;9(3):211-224

[26]. Rasgon NL et al. Depression in women with polycystic ovary syndrome: clinical and biochemical correlates.Journalof Affective Disorders.2003May;74(3):299-304.

[27]. Turhan NO et al.Assessment of glucose tolerance and pregnancy outcome of polycystic ovary patients. InternationalJournal of Gynecology \&Obstetrics.2003May;81(2):163-168.

[28]. Hoeger KM. Role of lifestyle modification in the management of polycystic ovary syndrome. Best Pract Res Clin Endocrinol Metab 2006;20:293-310.

[29]. Barnard L, Ferriday D, Guenther N, Strauss B, Balen AH, Dye L. Quality of life and psychological well being in polycystic ovarysyndrome. Human Reproduction, (2007).22:2279-2286.

[30]. Diamanti- Kandarakis E, Kouli CR, Bergiele AT, et al. A survey of the polycystic ovary syndrome in the Greek Island of Lesbos: hormonal and metabolic profile. J Clin Endocrinal Metab 1999;84:4006-4011.

[31]. Cipkala-Gaffin, J., Talbott, E. O., Song, M., Bromberger, J., \& Wilson, J. Associations between psychologic symptoms and life satisfaction in women with polycystic ovary syndrome. Journal Of Women's Health (15409996), (2012), 21(2), $179-187$.

[32]. Asunction M, Calvo RM, San Millian JL, et al. A prospective study of the prevalence of polycystic ovary syndrome in unselected Caucasian women from Spain. J Clin Endocrinol Metab 2000;85:2434- 2438.

[33]. Ching, H. L., Burke, V. V., \& Stuckey, B. A. Quality of life and psychological morbidity in women with polycystic ovary syndrome: body mass index, age and the provision of patient information are significant modifiers. Clinical Endocrinology, (2007) 66(3), 373-379.

[34]. Shobha (2014) An exploratory survey to identify the adolescents with high risk for Polycystic Ovarian Syndrome (PCOS) and to find the effectiveness of an awareness programme among students of selected pre university colleges of Udupi District IOSR Journal of ursing and Health Science (IOSR-JNHS) e-ISSN: 2320-1959.p- ISSN: 2320-1940 Volume 3, Issue 3 Ver. II , PP 6669.

[35]. March WA, Moore VM, Willson KJ, Phillips DI, Norman RJ, Davies MJ,2010 Prevalence of Polycystic Ovary Syndrome in a community sample done under contrasting diagnostic criteria. Hum Reprod;25:544-51.

[36]. Balen AH, Conway GS, Kaltsas G, Techatrasak K, Manning PJ, West C et al. Polycystic ovary syndrome: the spectrum of the disorder in 1741 patients. Hum Reprod 1995; 10(8):2107-2111. 